\title{
Design of the crashworthy structure of an urban aircraft
}

\author{
Bairong Shang, Jingwu $\mathrm{He}^{\mathrm{a}}$, Yuexi Xiong and Zhe $\mathrm{Hu}$ \\ School of Aeronautic Science and Engineering, Beihang University, Beijing, China
}

\begin{abstract}
With the development of general aviation, the urban aircraft is around the corner. The urban aircraft with composite is considered as an ultralight vehicle and the crashworthiness is of vital importance for such an ultralight aircraft. Composites are being widely and increasingly used in the aerospace industry because of their advantages that include the high specific strength and stiffness over traditional metallic materials. Besides, composites have the potential for absorbing the energy in a crash event. The crashworthiness of the cockpit section is analyzed in this paper and some modifications in the subfloor have been made to improve the survivability of the pilot. Advances in commercial softwares have enabled engineers to simulate crash events. The three-dimensional structure model is established by use of CATIA software and the crash process is simulated by MSC/DYTRAN. By comparing the crashworthiness of composite structures, reliable basis is provided for the design of a safe and sound structure.
\end{abstract}

\section{Introduction}

The crashworthiness is the ability to protect the safety of crew members and passengers during an aircraft crash accident and the aircraft accidents are severe threats to the life safety of people. With the development of the civil aviation scientific technology, more and more attention is attracted by the crashworthiness of civil aircraft [1]. The airplane's crash is an uncertain process and the true process of impact is also very complicated, therefore the reasonable crash condition and crash environment is the precondition to acquire an accurate structural response [2].Two methods are mainly used in the research on crashworthiness of aircraft structure, the drop impact test and the finite element simulation. The drop impact test needs a lot of time and costs. Moreover, the accuracy and reliability of test data depend much on sensor elements and data collection methods. However, the crash simulation method can greatly shorten the research cycle and reduce the costs. So a reasonable crash simulation will raise the design efficiency and give designers a comprehensive understanding of the characteristics of the crash.

An increasing number of research institutions have studied the crashworthiness of the fight vehicle. Back in the 1970s, Federal Aviation Administration and National Aeronautics and Space Administration started the drop impact test of aircrafts, including small aircraft and large aircraft supported by industry departments of the United States. Meanwhile, extensive research and analysis

\footnotetext{
${ }^{a}$ Corresponding author : hejingwu@buaa.edu.cn
} 
on the dynamic characteristics of aircraft crash and the safety were carried out [3]. In 1999, NASA Langley Research Center conducted a crash test of the Sikorsky ACAP helicopter and simulated the crash process by the finite element method, aiming at verifying the validity of the finite element simulation. The simulation was by use of the software MSC.Dytran and the results of the simulation and crash test were similar. So it proved that the finite element simulation method is feasible in crash resistance design [4].

In the past 50 years, composites have been used in the aircraft structure gradually and a great deal of work about the failure behavior of composites has been done. In the 1990s, the European Union organized a project entitled "CRASHWORTHINESS" which acquired the crash test data of a fullscale fuselage. Then a series study of composite structure about crash resistance were carried on, which were emphasized on carbon fiber reinforced composite material characteristics and failure mechanism [5-7].

The study of the crashworthy structure mainly contains seat systems, landing gear systems and fuselage structure $[8,9]$. The method of the crashworthy structure design is different among the light fixed-wing aircraft, the helicopter and the large and medium-sized aircraft [10]. Due to constrained space under the floor in the light fixed-wing aircraft and helicopter, the subfloor of energy absorption is widely used to dissipate the kinetic energy during the impact. However, it is focused on the struts and reinforced bulkheads in the large and medium-sized aircraft and the reinforced bulkheads are the important structure to absorb the kinetic energy. The cross-sections are respectively shown in Figure 1 and Figure 2.

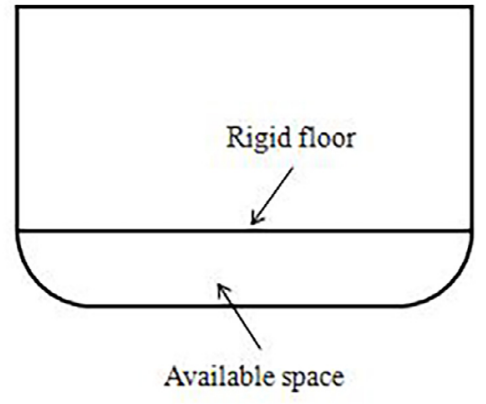

Figure 1. Layout of the light fixed-wing aircraft and helicopter.

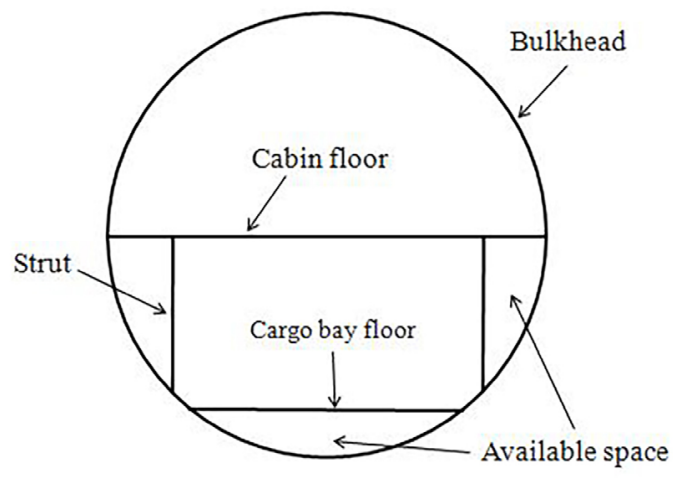

Figure 2. Layout of the large and medium-sized aircraft.

\section{Energy analysis during the crash process}

In the process of aircraft crash, by the law of conservation of energy

$$
\frac{1}{2} M\left(V_{0}^{2}-V_{1}^{2}\right)=U_{G}+U_{F}
$$

Where $M$ is the quality of the aircraft, $V_{0}$ is the initial velocity when contact the ground, $V_{l}$ is the velocity when fuselage rebound, $U_{G}$ is the energy absorbed by the ground and $U_{F}$ is the energy absorbed by the fuselage structure.

$U_{F}$ will meet the equation

$$
U_{F}=U_{\text {cabin }}+U_{\text {subfloor }}
$$

Where $U_{\text {cabin }}$ is the energy absorbed during the cabin destruction and $U_{\text {subfloor }}$ is the energy absorbed during the subfloor destruction 
According to the equation (1) and equation (2), $U_{\text {cabin }}$ will meet the equation

$$
U_{\text {cabin }}=\left[\frac{1}{2} M\left(V_{0}^{2}-V_{1}^{2}\right)-U_{G}\right]-U_{\text {subfloor }}
$$

In order to maintain people's survival space and reduce overload, the equation indicates:

1) Try to reduce the impact velocity and it requires that the pilot have to control the descent velocity and attitude reasonably. But this is not the emphasis in this paper.

2) Try to increase the extent of the damage of subfloor structure which helps to absorb the kinetic energy as much as possible

\section{Model of the unmodified cockpit section}

Because of the development of general aviation and the urban traffic jam, the urban aircraft is around the corner. The security must be the priority for the ultralight aircraft. A vertical drop of the cockpit section, which crashes vertically to impact at $8 \mathrm{~m} / \mathrm{s}$ is simulated in this paper. The cockpit section is approximately $1200 \mathrm{~mm}$ in length and $800 \mathrm{~mm}$ in maximum width. The structure of the aircraft is mainly composite structure and the three-dimensional structure model is established in the software CATIA, shown in Figure 3 and Figure 4. The main structure of the cockpit section are the skin, the bulkheads, the stringers and the floor. The crossbeams and the bulkheads, supporting the floor panel are integrated together. There are stiffeners below the floor panel as well. In order to attain enough stiffness and strength and reduce weight, Carbon Fiber Reinforced Plastic (CFRP) is chosen and T300 carbon fabric is used in the whole cockpit section.

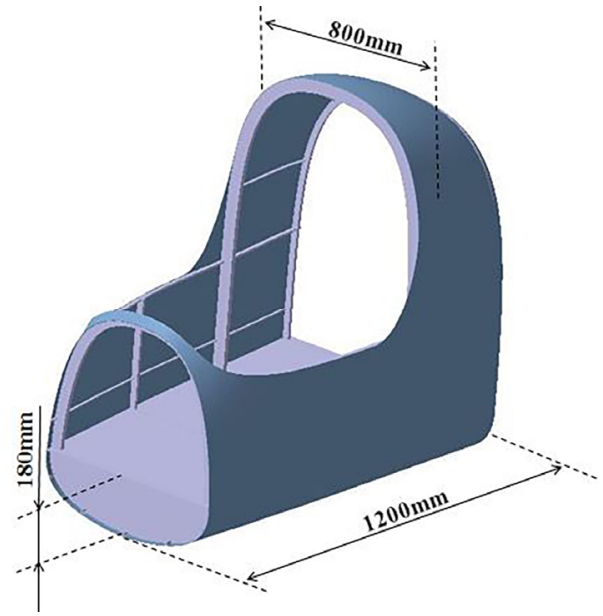

Figure 3. Structural model of the cockpit section.

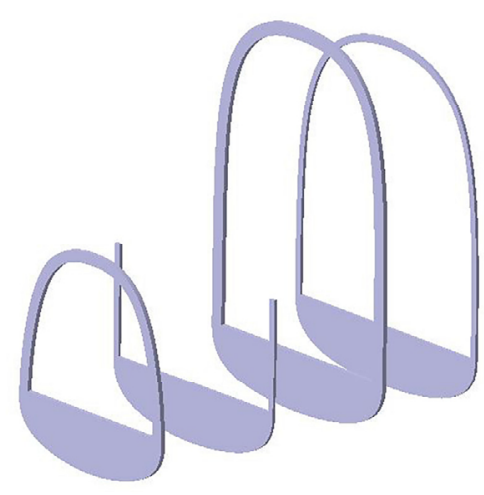

Figure 4. Structural model of bulkheads.

The unmodified cockpit section is simplified and the finite model is established. First ,the mesh is completed in the software Hypermesh and then the model is imported into the software Patran where the loads, the boundary conditions and the output requirements are set up. At last, the simulation is operated in the solver Dytran. According to the standards about the crashworthiness, the vertical impact velocity is set as $8 \mathrm{~m} / \mathrm{s}$ and the ground is simplified as a rigid plane. The direction of the impact is vertical to the rigid plane. Furthermore, the pilot and the seat are simplified as a mass element which is connected to the floor and the connections in the model are regarded as rigid connections. The finite model is shown in Figure 5, Figure 6 and Figure7. The floor panel is a composite sandwich construction with a foam core and T300 carbon fabric face sheets, shown in Figure 8. The property of T300 and other materials is shown in Table 1 and Table 2.The whole model is meshed with shell elements and consists of 33209 nodes, 32837 elements and one concentrated mass element. The 
explicit algorithms for structural dynamic analysis is adopted to solve the impact problem. The structural mass of the cockpit section is $11.94 \mathrm{~kg}$. There are a few contact constraints in the finite model, in consequence it will cost several hours to complete the simulation.

Table 1. Material property data of T300.

\begin{tabular}{|c|c|c|c|c|c|c|c|c|c|}
\hline $\begin{array}{c}\rho \\
\left(\mathrm{g} / \mathrm{cm}^{3}\right)\end{array}$ & $v$ & $\begin{array}{c}\mathrm{G}_{12} \\
(\mathrm{GPa})\end{array}$ & $\begin{array}{c}\mathrm{E}_{11} \\
(\mathrm{GPa})\end{array}$ & $\begin{array}{c}\mathrm{E}_{22} \\
(\mathrm{GPa})\end{array}$ & $\begin{array}{c}\mathrm{X}_{\mathrm{T}} \\
(\mathrm{MPa})\end{array}$ & $\begin{array}{c}\mathrm{X}_{\mathrm{C}} \\
(\mathrm{MPa})\end{array}$ & $\begin{array}{c}\mathrm{Y}_{\mathrm{T}} \\
(\mathrm{MPa})\end{array}$ & $\begin{array}{c}\mathrm{Y}_{\mathrm{C}} \\
(\mathrm{MPa})\end{array}$ & $\begin{array}{c}\mathrm{S} \\
(\mathrm{MPa})\end{array}$ \\
\hline 1.57 & 0.33 & 5.0 & 135 & 9.75 & 1560 & 1226 & 80 & 218 & 89.9 \\
\hline
\end{tabular}

Table 2. Other material property data used in the finite model.

\begin{tabular}{|c|c|c|c|c|c|c|}
\hline Material & $\begin{array}{c}\rho \\
\left(\mathrm{g} / \mathrm{cm}^{3}\right)\end{array}$ & $\begin{array}{c}\mathrm{E} \\
(\mathrm{MPa})\end{array}$ & $v$ & $\begin{array}{c}\sigma_{\mathrm{y}} \\
(\mathrm{MPa})\end{array}$ & $\begin{array}{c}\mathrm{E}_{\mathrm{h}} \\
(\mathrm{MPa})\end{array}$ & $\varepsilon_{\mathrm{psf}}$ \\
\hline Rohacell foam & 0.045 & 13.78 & 0.3 & 0.62 & 0.37 & 0.8 \\
\hline Foam 1 & 0.052 & 75 & 0.38 & 1.6 & & 0.03 \\
\hline $0^{\circ} / 90^{\circ}$ E-glass & 0.24 & 18495 & 0.13 & 85 & 813 & 0.01 \\
\hline $\pm 45^{\circ}$ E-glass & 0.24 & 10360 & 0.47 & 63 & 813 & 0.01 \\
\hline
\end{tabular}

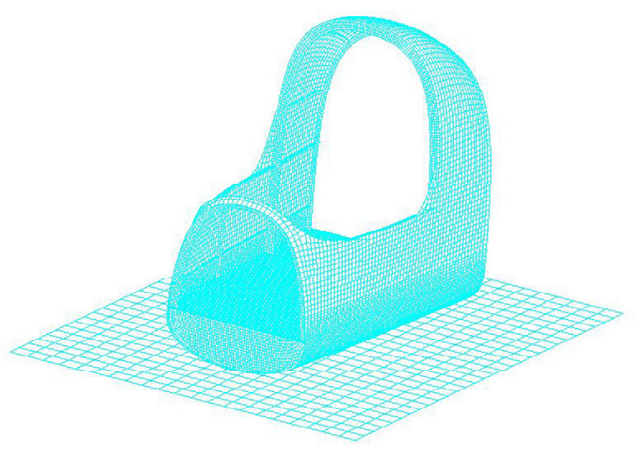

Figure 5. Finite model of the unmodified cockpit section.

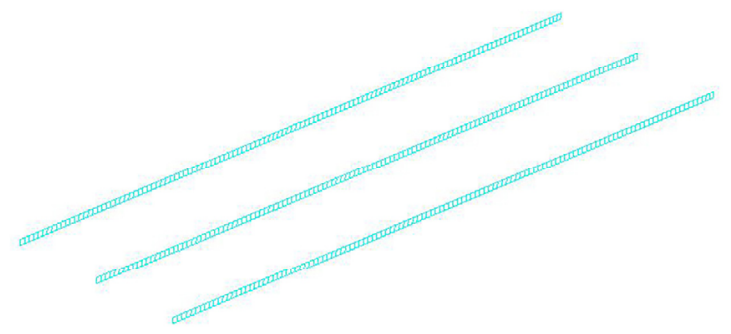

Figure 7. Finite model of the floor stiffeners.

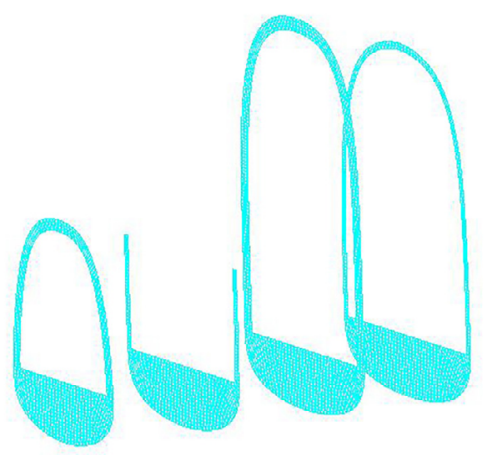

Figure 6. Finite model of the bulkheads.

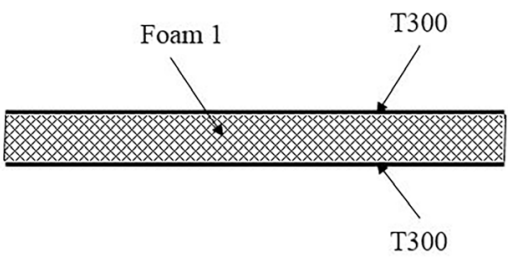

Figure 8. Schematic drawing of the floor panel.

\section{Simulation results of the unmodified cockpit section}

The peak value of acceleration, appearing at the time $2.2 \mathrm{~ms}$ is $364.6 \mathrm{~g}$, shown in Figure 10 . The peak acceleration appears at the time when the cockpit section just contacts to the rigid plane which indicates that the strength and stiffness of the structure are too high. Then with the destruction of the structure and the energy absorption, the acceleration decreases gradually. From Figure 11, it is easy to figure out that the cockpit section rebounds at $6.5 \mathrm{~ms}$. As can be seen from Figure 9, only a little part 
has deformed and damaged, and the whole section maintains the intact structure. Although the survival space is enough, the peak acceleration is as high as $364.6 \mathrm{~g}$. The overload does sever harm to people's body, so it requires that the stiffness and strength have to be reduced so that the structure will damage further and the kinetic energy will be absorbed step by step, which will contribute to reduce the peak acceleration.

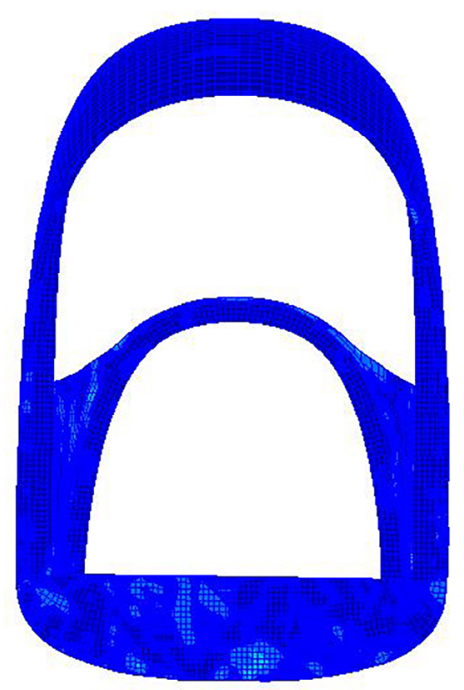

Figure 9. Deformation of the unmodified cockpit section at $6.5 \mathrm{~ms}$.

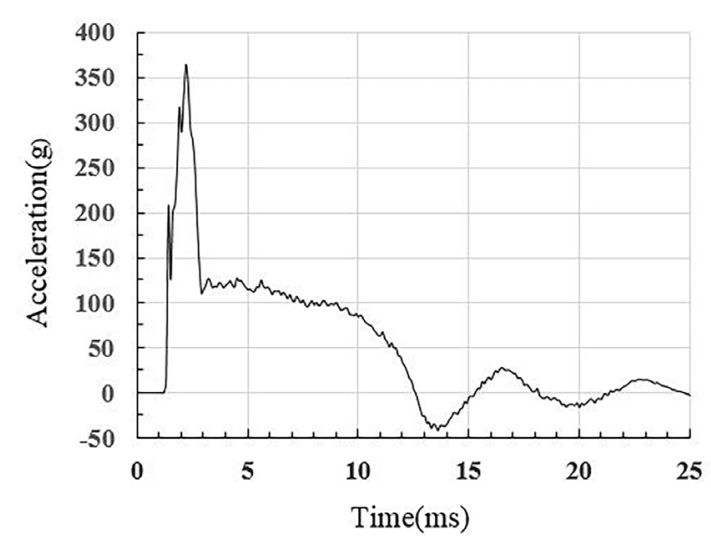

Figure 10.Time history of acceleration.

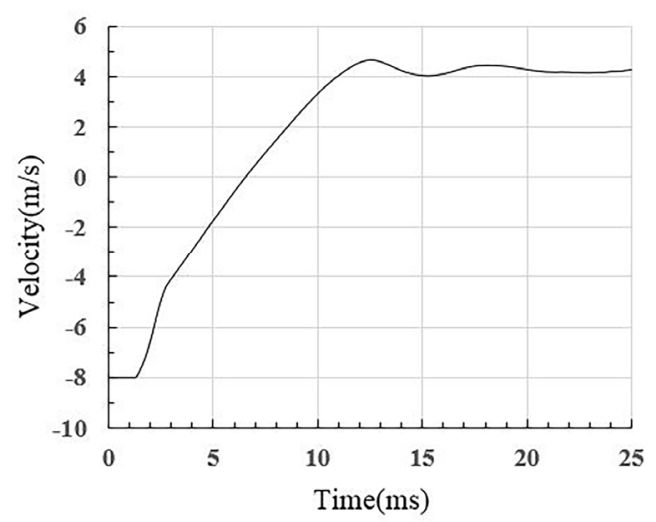

Figure 11. Time history of velocity.

\section{Improvement of the subfloor}

The Rohacell foam that has good energy-absorbing performance is added at the bottom and the bulkheads and some auxiliary structure are added to support the floor. In addition, the beams supporting the floor panel have also been modified.The finite models are shown in Figure 14, Figure 15 and Figure 16. In order to make the upper structure of the cockpit section stiff enough, the top part of the skin, the bulkheads and the auxiliary structure are carbon fiber reinforced plastic structure and the lower part are glass fiber reinforced plastic structure which is easier to damage and absorb the energy. The material distribution is shown in Figure 12 and Figure 13 and T300 and E-galss fabric are used in the model. The structural mass of the cockpit section is $10.72 \mathrm{~kg}$. 

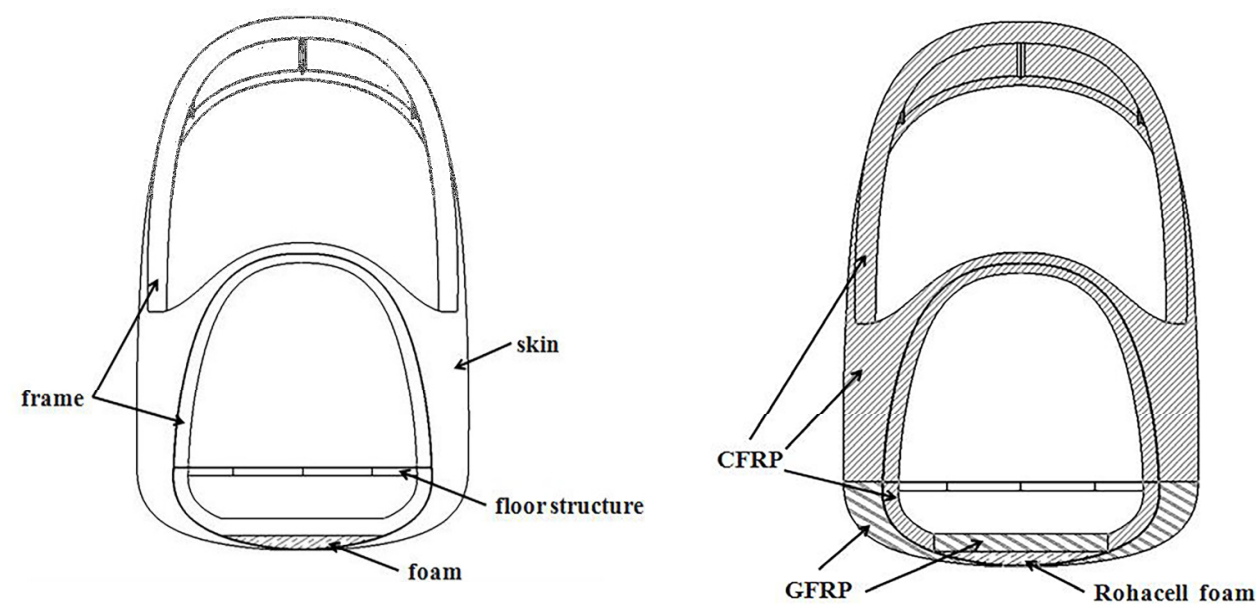

Figure 12. Front view of the modified cockpit section. Figure 13.Schematic drawing of material distribution.

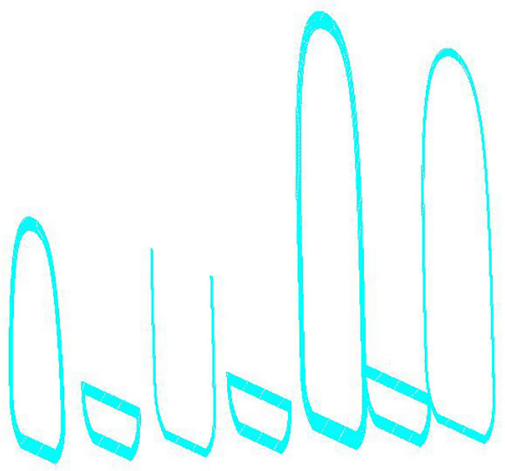

Figure 14. Finite model of the modified bulkheads and the auxiliary structure.

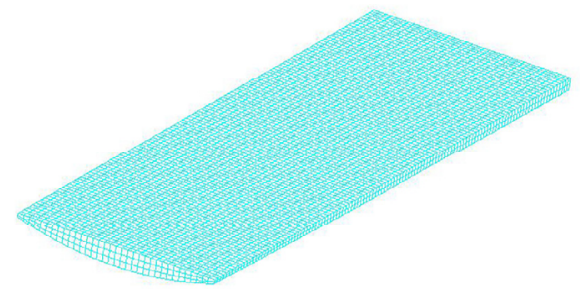

Figure 15. Finite model of the foam.

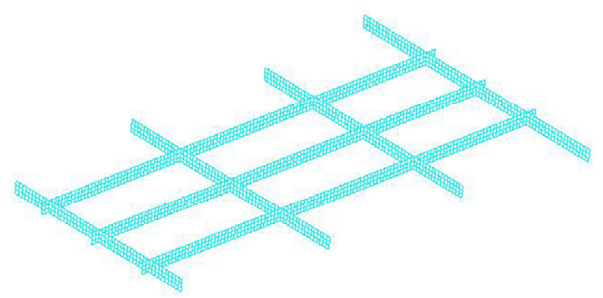

Figure 16. Beams of the floor structure.

As can be seen from Figure 17, the subfloor has damaged badly and been crushing. For the sake of reducing the acceleration when the cockpit section contacts the ground, firstly the added foam at the bottom is compressed to absorb the energy. Secondly, the plastic deformation and the destruction occurs in the glass fiber reinforced plastic. Finally, the subfloor with CFRP is destroyed gradually. This multi-level buffering structure makes the process of energy absorption slow and steady. The peak acceleration is about 55.2g, shown in Figure 18. It can been seen from Figure 19 that the cockpit section rebounds at nearly $28.9 \mathrm{~ms}$ and remains at the velocity of $2.6 \mathrm{~m} / \mathrm{s}$ at $60 \mathrm{~ms}$.

Compared with the result of the unmodified cockpit section, the peak acceleration obviously decreased by $84.9 \%$ and the acceleration remains in a stable range, shown in Figure 20. In addition, the upper cockpit section which has a little deformation and destruction maintains an enough survival 
space. Therefore the chance of the pilot's survival has been greatly increased. The mass of the modified cockpit section is $1.22 \mathrm{~kg}$ less than the unmodified one, which will conrtibute to improve the flight performance.

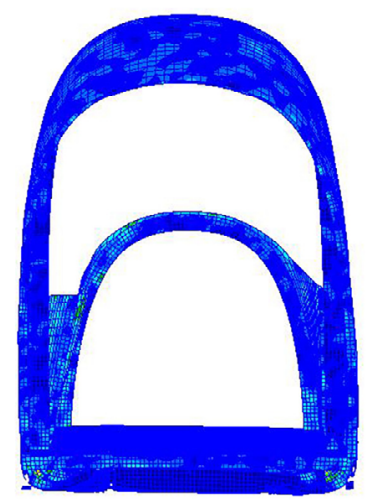

Figure 17. Deformation of the modified cockpit section at $28.9 \mathrm{~ms}$.

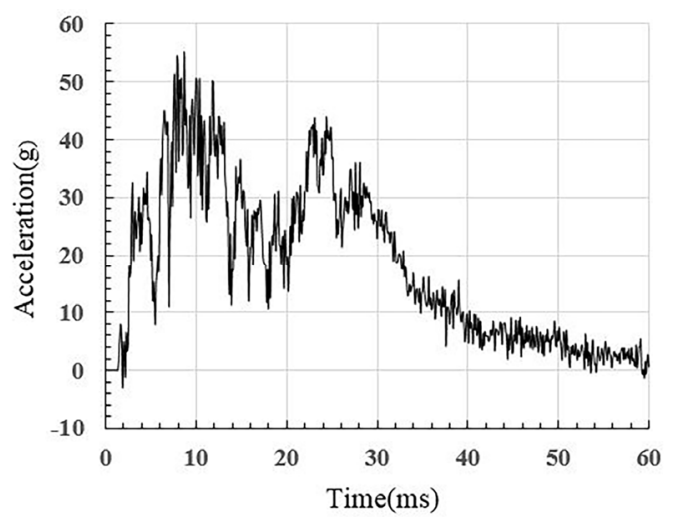

Figure 18. Time history of acceleration for the modified cockpit section.

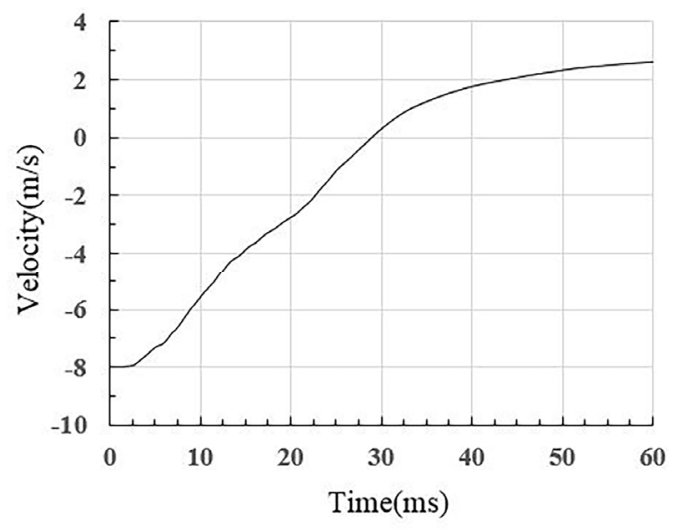

Figure 19. Time history of velocity for the modified cockpit section.

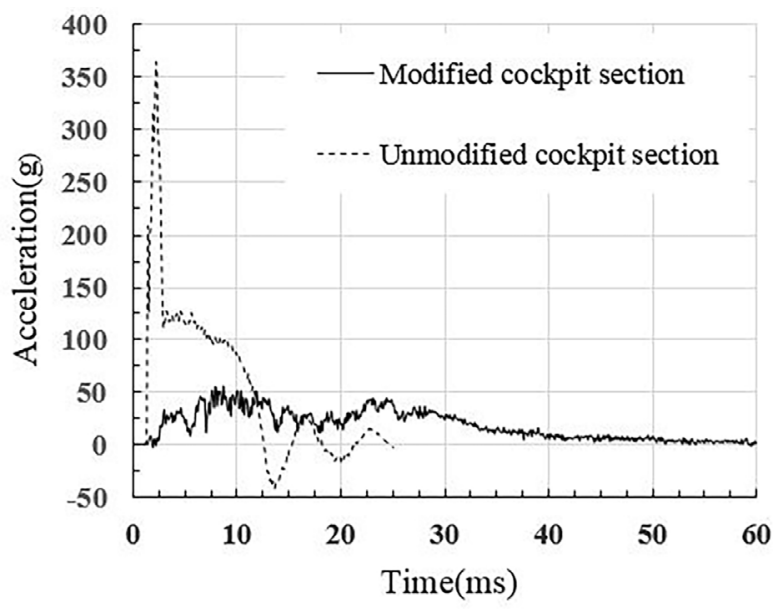

Figure 20. Comparison of acceleration histories. 


\section{Summary}

The crashworthiness of aircraft is of vital importance and a crash simulation of the composite cockpit section is simulated in the transient dynamic finite element software MSC.Dytran. Because of the high specific strength and specific stiffness, T300 carbon fabric is used in the structure. But the stiffness and strength of the unmodified cockpit section is so high that the high impact loads transmits to the occupant. In order to limit the loads transmitted to the seat, the Rohacell foam and E-glass fabric are used at the bottom of the modified cockpit section. The progressive failure of the subfloor makes the kinetic energy absorbed step by step and reduces the loads transmitted to the seat. Compared with the peak acceleration of the unmodified cockpit section, the peak acceleration of the modified one is reduced by $80 \%$ and the mass reduced by $10.2 \%$. The Rohacell foam and E-glass help to absorb the energy during the crash and the progressive failure of the subfloor contributes to make the acceleration remain in a stable range. The design concept for the subfloor of the ultralight, introduced in this paper has certain significance.

\section{References}

1. H. Zhang, Design guideline for crash resistance of the general aircraft (2009)

2. Z.Y. Feng, X.M. Zhang, H.L. Mou, and T. C. Zou, MSTAE, 32, 353 (2013)

3. E.L. Fasanella and K.E. Jackson, Proceedings of the 44th AIAA Structures, Structural Dynamics and Materials Conference (2003)

4. E.L. Fasanella, K.E. Jackson, and K.H. Lyle, J. Am. Helicopter Soc., 47, 156-168 (2002)

5. G. Labeas and T. Kermanidis, FFEMS, 26, 449-457 (2003)

6. E. Deletombe and D. Delsart, Aerosp. Sci. Technol., 4,189-199 (2000)

7. J.F.M. Wiggenraad, D. Santoro, F. Lepage, C. Kindervater, and H.C. Mañez, The 57th Annual Forum of the American Helicopter Society (2001)

8. A.O. Bolukbasi and D.H. Laananen, J. Aircraft, 21, 512-519 (1984)

9. K.H. Lyle, K.E. Jackson, and E.L. Fasanella, J. Aircraft, 39, 142-147 (2002)

10. J.D. Cronkhite and V.L. Berry, NASA Contractor Report No.3603 (1982) 